\title{
Nonlinear Ridge Regression Improves Robustness of Cell-Type-Specific Differential Expression Studies
}

Fumihiko Takeuchi ( $\nabla$ fumihiko@takeuchi.name)

Kokuritsu Kenkyu Kaihatsu Hojin Kokuritsu Kokusai Iryo Kenkyu Center https://orcid.org/0000-00033185-5661

\section{Norihiro Kato}

Kokuritsu Kenkyu Kaihatsu Hojin Kokuritsu Kokusai Iryo Kenkyu Center

\section{Methodology article}

Keywords: Epigenome-wide association study, Differential gene expression analysis, Cell type, Ridge regression, Nonlinear regression, $\mathrm{mQTL}, \mathrm{eQTL}$

Posted Date: July 1st, 2020

DOl: https://doi.org/10.21203/rs.3.rs-39226/v1

License: (9) This work is licensed under a Creative Commons Attribution 4.0 International License. Read Full License

Version of Record: A version of this preprint was published on March 22nd, 2021. See the published version at https://doi.org/10.1186/s12859-021-03982-3. 


\section{Nonlinear ridge regression improves robustness of cell-type-specific differential expression studies}

Fumihiko Takeuchi and Norihiro Kato

Department of Gene Diagnostics and Therapeutics, Research Institute, National Center for Global Health and Medicine (NCGM), Tokyo, Japan

\section{Correspondence}

Fumihiko Takeuchi

Department of Gene Diagnostics and Therapeutics, Research Institute, National Center for Global Health and Medicine (NCGM)

1-21-1 Toyama, Shinjuku-ku, Tokyo, 162-8655, Japan

Email: fumihiko@takeuchi.name 


\section{Abstract}

2 Background: Epigenome-wide association studies (EWAS) and differential 3 gene expression analyses are generally performed on tissue samples, which 4 consist of multiple cell types. Cell-type-specific effects of a trait, such as 5 disease, on the omics expression are of interest but difficult or costly to 6 measure experimentally. By measuring omics data for the bulk tissue, cell 7 type composition of a sample can be inferred statistically. Subsequently, cell8 type-specific effects are estimated by linear regression that includes terms 9 representing the interaction between the cell type proportions and the trait. 10 This approach involves two issues, scaling and multicollinearity.

11 Results: First, although cell composition is analyzed in linear scale, 12 differential methylation/expression is analyzed suitably in the logit/log scale. 13 To simultaneously analyze two scales, we developed nonlinear regression. 14 Second, we show that the interaction terms are highly collinear, which is 15 obstructive to ordinary regression. To cope with the multicollinearity, we 16 applied ridge regularization. In simulated and real data, the improvement was 17 modest by nonlinear regression and substantial by ridge regularization. 18 Conclusion: Nonlinear ridge regression performed cell-type-specific 19 association test on bulk omics data more robustly than previous methods. 20 The omicwas package for $\mathrm{R}$ implements nonlinear ridge regression for cell21 type-specific EWAS, differential gene expression and QTL analyses. The 22 software is freely available from https://github.com/fumi-github/omicwas

\section{Keywords}

25 Epigenome-wide association study, Differential gene expression analysis, Cell 26 type, Ridge regression, Nonlinear regression, mQTL, eQTL 


\section{Background}

29 Epigenome-wide association studies (EWAS) and differential gene expression 30 analyses elucidate the association of disease traits (or conditions) with the 31 level of omics expression, namely DNA methylation and gene expression. 32 Thus far, tissue samples, which consist of heterogeneous cell types, have 33 mainly been examined, because cell sorting is not feasible in most tissues 34 and single-cell assay is still expensive. Nevertheless, the cell type composition of a sample can be quantified statistically by comparing omics measurement of the target sample with reference data obtained from sorted or single cells $[1,2]$. By utilizing the composition, the disease association specific to a cell type was statistically inferred for gene expression [3-10] and DNA methylation [11-14].

For the imputation of cell type composition, omics markers are usually analyzed in the original linear scale, which measures the proportion of mRNA molecules from a specific gene or the proportion of methylated cytosine molecules among all cytosines at a specific CpG site [15]. The proportion can differ between cell types, and the weighted average of cell-type-specific proportions becomes the proportion in a bulk tissue sample. Using the fact that the weight equals the cell type composition, the cell type composition of a sample is imputed. In contrast, gene expression analyses are performed in the log-transformed scale because the signal and noise are normally distributed after log-transformation [16]. In DNA methylation analysis, the logit-transformed scale, which is called the M-value, is statistically valid [17].

51 Consequently, the optimal scales for analyzing differential gene expression 52 or methylation can differ from the optimal scale for analyzing cell type 53 composition.

54 Aiming to perform cell-type-specific EWAS or differential gene expression 55 analyses by using unsorted tissue samples, we study two issues that have been overlooked. Whereas previous studies were performed in linear scale, we develop a nonlinear regression, which simultaneously analyzes cell type composition in linear scale and differential expression/methylation in log/logit scale. The second issue is multicollinearity. Cell-type-specific effects of a trait, such as disease, on omics expression are usually estimated by linear 
61 regression that includes terms representing the interaction between the cell

62 type proportions and the trait. We show that the interaction terms can

63 mutually be highly correlated, which obstructs ordinary regression. To cope 64 with the multicollinearity, we implement ridge regularization. Our methods 65 and previous ones are compared in simulated and real data.

\section{Results}

\section{Multicollinearity of interaction terms}

69 Typically, cell-type-specific effects of a trait on omics marker expression is analyzed by the linear regression in equation (2). The goal is to estimate $\beta_{h, k}$, the effect of trait $k$ on the expression level in cell type $h$. This is estimated

72 based on the relation between the bulk expression level $Y_{i}$ of a sample and 73 the regressor $W_{h, i} X_{i, k}$, which is an interaction term defined as the product of 74 the cell type proportion $W_{h, i}$ and the trait value $X_{i, k}$ of the sample. The 75 variable $W_{h}$ for cell type composition cannot be mean-centered, and 76 interaction terms involving uncentered variables cause multicollinearity [18].

77 We first survey the extent of multicollinearity in real data for cell-type-specific 78 association.

In peripheral blood leukocyte data from a rheumatoid arthritis study 80 (GSE42861), the proportion of cell types ranged from 0.59 for neutrophils to 810.01 for eosinophils (Table $1 \mathrm{~A}$ ). The proportion of neutrophils was negatively 82 correlated with the proportion of other cell types (apart from monocytes) with 83 correlation coefficient of -0.68 to -0.46 , whereas the correlation was weaker 84 for other pairs (Table 1B). Rheumatoid arthritis status was modestly correlated with proportions of cell types. The product of the disease status $X_{k}$, centered to have zero mean, and the proportion of a cell type becomes

87 an interaction term. The correlation coefficients between the interaction terms are mostly $>0.8$, apart from eosinophils (Table $1 \mathrm{C}$ ). The ratio of mean to SD of the proportion is high for all cell types apart from eosinophils (Table $1 \mathrm{~A})$. The interaction terms for high-ratio cell types are strongly correlated 
91 with $X_{k}$, which in turn causes strong correlation between the relevant 92 interaction terms.

93 The situation was the same for the interaction with age in GTEx data. The 94 granulocytes (which include neutrophils and eosinophils) were the most 95 abundant (Table 2A). The proportion of granulocytes was negatively 96 correlated with other cell types (apart from monocytes) with correlation 97 coefficient of -0.89 to -0.41 , and the correlation between other pairs was 98 generally weaker (Table 2B). Age was modestly correlated with proportions 99 of cell types. In this dataset, the ratio of mean to SD of the proportion was 100 high in all cell types (Table 2A), which caused strong mutual correlation 101 between interaction terms (Table 2C).

102 In the above empirical data, multicollinearity between interaction terms 103 seemed to arise not due to the correlation between cell type proportions or $X_{k}$, but due to the high ratio of mean to SD in the cell type proportions. Subsequently, this property was derived mathematically. As we derived in equation (17), the correlation between interaction terms $W_{h} X_{k}$ and $W_{h^{\prime}} X_{k}$ approaches to one, when the ratios $\mathrm{E}\left[W_{h}\right] / \operatorname{SD}\left[W_{h}\right]$ and $\mathrm{E}\left[W_{h^{\prime}}\right] / \operatorname{SD}\left[W_{h^{\prime}}\right]$ are high, irrespective of $\operatorname{Cor}\left[W_{h}, W_{h^{\prime}}\right]$ (Figure 1 ). The ratio was 1.6 to 5.3 (apart from eosinophils) in the rheumatoid arthritis dataset and $\geq 4.3$ in the GTEX dataset. We looked up datasets of several ethnicities and found the ratio to

111 be $\geq 1.5$ in majority of cell types (Additional file 1: Table S1). Thus,

112 multicollinearity can be a common problem for cell-type-specific association 113 analyses.

\section{Evaluation in simulated data}

115 By using simulated data, we evaluated previous methods and new 116 approaches of the omicwas package. In order to simultaneously analyze two 117 scales, the linear scale for heterogeneous cell mixing and the log/logit scale 118 for trait effects, we applied nonlinear regression in omicwas (equations (4) 119 and (5)). To cope with the multicollinearity of interaction terms, we applied 120 ridge regularization (equations (9) and (10)).

121 Previous regression type methods are based either on the full model of 122 linear regression (equation (2)) or the marginal model (equation (3)). The 
123 full model fits and tests cell-type-specific effects for all cell types

124 simultaneously, and its derivatives include TOAST, cSSAM.Im,

125 CellDMC.unfiltered and CellDMC.filtered. The marginal model fits and tests

126 cell-type-specific effect for one cell type at a time, and its derivatives include

127 csSAM.monovariate and TCA.

128 The simulation data was generated from real datasets of DNA methylation

129 and gene expression. The original cell type composition was retained for all 130 samples, and the case-control status was randomly assigned. In each sample, 131 expression level in each cell type was randomly determined according to a 132 scenario, and then averaged according to the sample's cell type composition. 133 Under each statistical algorithm, the disease association in the target cell type was assessed by a Z-score, comparing cases vs controls.

In scenario A for DNA methylation, expression of all cell types had identical distribution, irrespective of the case/control status (Figure 2A). The

137 type I error rate was controlled ( $\leq 0.05)$ in all algorithms. In scenario $B$, cases 138 had higher expression level in one randomly selected cell type, and that cell 139 type was tested (Figure 2B). Here, the most appropriate algorithm is the 140 marginal test applied to the perturbed cell type, which indeed attained the 141 highest power. For the most abundant neutrophils, the Z-score was in the 142 high range of 9.9 to 14.9 for the marginal test. With regards to the power, 143 the ridge regression methods (omicwas.identity.ridge and 144 omicwas.logit.ridge) came next. The algorithms based on full model, without 145 ridge regularization, (Full, TOAST, CellDMC.unfiltered, omicwas.identity and 146 omicwas.logit) gained modest power. TCA, which is similar to the marginal 147 test, detected neutrophil-specific association with high Z-score, but the power 148 over all cell types was modest. In scenario $C$, the expression level of cases 149 was lower in one cell type, which was not the tested cell type (Figure 2C). 150 Since the expression of the tested cell type is identical between cases and 151 controls, a correct algorithm should detect no signal. The type I error rate 152 was inflated, being highest for the marginal test, followed by the ridge 153 regression methods and TCA. Extremely strong spurious signals of Z-score < $154-6$ were detected in marginal and TCA. Scenario D combined scenarios B and 155 C, where the tested cell type had higher expression in cases, and one non- 
156 tested cell type had lower expression in cases (Figure 2D). The distribution

157 of neutrophil Z-score was similar to scenario B, and the spurious signals with 158 low Z-scores were similar to scenario C. Over all scenarios, the similarity in 159 performance of omicwas.identity vs omicwas.logit, as well as 160 omicwas.identity.ridge vs omicwas.logit.ridge, indicates that the scaling was 161 not influential in DNA methylation data.

162 The results for simulated gene expression data were similar. In scenario 163 A with no true signal, type I error rate was controlled $(\leq 0.05)$ in all algorithms 164 (Figure 3A). In scenario B, where true signal exists only for the tested cell 165 type, the power was the highest in marginal and relatively high in 166 csSAM.monovariate (Figure 3B). The power was in decreasing order, omicwas.log.ridge $>$ omicwas.identity.ridge $>$ omicwas.log > omicwas.identity; proper scaling modestly improved performance. In scenario C, where cases have lower expression in one non-target cell type, the type I error was inflated in the negative direction, with the largest inflation

171 in marginal, and moderate inflation in ridge regression methods and 172 csSAM.monovariate (Figure 3C). Extremely strong false signals of Z-score < $173-6$ occurred in marginal and csSAM.monovariate. In scenario D, where the 174 tested cell type has higher expression in cases, while one non-tested cell type 175 has lower expression, we could observe the overlay of power gain of scenario 176 B and type I error inflation of scenario C (Figure 3D).

177 Although we roughly grouped previous algorithms into derivatives of full 178 or derivatives of marginal, some implement treatments beyond simple linear 179 models. For example, the TCA algorithm tends to detect neutrophil signals 180 similarly as the marginal test (Fig. 2B), yet had smaller type I error rate (Fig. $1812 \mathrm{C})$.

\section{Cell-type-specific association with rheumatoid arthritis and age}

183 The cell-type-specific association of DNA methylation with rheumatoid 184 arthritis was predicted using bulk peripheral blood leukocyte data and was 185 evaluated in sorted monocytes (Figure 4A) and B cells (Figure 4B). Whereas 186 the full model (and its derivatives) performed the best and the marginal 187 model (and its derivatives) performed the worst in monocytes, the 
performance ranking was opposite in B cells. A robust algorithm would consistently achieve high performance relative to the best algorithm in each instance. Nonlinear ridge regression (omicwas.logit.ridge) was the most robust, performing $65 \%$ to $93 \%$ relative to the best method.

The cell-type-specific association of gene expression with age was predicted using whole blood data and was evaluated in sorted CD4 ${ }^{+} \mathrm{T}$ cells

194 (Figure 4C) and monocytes (Figure 4D). All algorithms performed poorly in $195 \mathrm{CD}^{+} \mathrm{T}$ cells, and the marginal model performed the best in monocytes. 196 Overall, nonlinear ridge regression (omicwas.log.ridge) was next to the marginal model, performing $21 \%$ to $47 \%$ to the marginal.

For dataset GSE42861 and for GTEx whole blood, the omicwas.logit.ridge and omicwas.log.ridge models of the omicwas package was computed in 8.1 and 0.7 hours respectively, using 8 cores of a $2.5 \mathrm{GHz}$ Xeon CPU Linux server.

\section{Discussion}

203 Aiming to elucidate cell-type-specific trait association in DNA methylation and gene expression, this article explored two aspects, multicollinearity and scale. We observed multicollinearity in real data and derived mathematically how it emerges. To cope with the multicollinearity, we proposed ridge regression. To properly handle multiple scales simultaneously, we developed nonlinear regression. By testing in simulated and real data, we found proper scaling to modestly improve performance. In contrast, ridge regression achieved performance that was more robust than previous methods.

The statistical methods discussed in this article are applicable, in principle,

212 to any tissue. For validation of the methods, we need datasets for bulk tissue 213 as well as sorted cells, ideally of >100 samples. Currently, the publicly 214 available data is limited to peripheral blood. By no means, we claim the 215 rheumatoid arthritis EWAS datasets [19-21] or the datasets for age 216 association of gene expression $[22,23]$ to be representative. Nevertheless, 217 we think verification in real data is important, which has not been performed 218 previously in large sample size.

219 By the performance in simulated and real data, we can roughly divide 
220 algorithms into three groups: full (and its derivatives), marginal (and its

221 derivatives) and ridge models. In marginal models, we test one cell type at a

222 time. If we knew in advance that one particular cell type is associated with

223 the trait, which would be a rare situation, testing that cell type in the marginal

224 model is the most simple and correct approach. Indeed, under such a

225 simulated scenario, the marginal test attained highest power (Figs. 2B, 3B).

226 However, when the test target cell type is not associated, but instead another

227 cell type is associated, the marginal tests can pick up false signals due to the

228 collinearity between regressor variables (Figs. 2C, 3C). The high power and

229 high error rate of the marginal tests can lead to unstable performance; in real

230 data, the marginal tests were the most powerful for detecting $B$ cell specific

231 association with rheumatoid arthritis (Fig. 4B) but were the least powerful for

232 monocytes (Fig. 4A). The full model tests all cell types together, and its

233 performance was the opposite of the marginal. By fitting all cell types

234 simultaneously, the full model adjusts for the effects of other cell types. The

235 full models did not detect false association coming indirectly from non-target

236 cell types (Figs. 2C, 3C), yet their power was relatively low (Figs. 2B, 3B).

237 The ridge tests (omicwas.identity.ridge, omicwas.logit.ridge and

238 omicwas.log.ridge) were in the middle between full and marginal tests with

239 regards to the power (Figs. 2B, 3B, 4). The false positives of ridge tests were

240 modest compared to the marginal tests (Figs. 2C, 3C).

241 We mathematically modeled and implemented the logit scale for DNA

242 methylation and log scale for gene expression. It turns out that the

243 improvement by formulating the nonlinear scale was negligible for DNA

244 methylation (Fig. 2B) and modest for gene expression (Fig. 3B;

245 omicwas.identity vs omicwas.log, and omicwas.identity.ridge vs

246 omicwas.log.ridge). This implies that previous works, which were almost

247 exclusively in linear scale, were not losing much power due to scaling.

\section{Conclusions}

250 For cell-type-specific differential expression analysis by using unsorted tissue 251 samples, we recommend trying ridge regression as a first choice because it 
252 balances power and type I error. Although marginal tests can be powerful 253 when the tested cell type actually is the only one associated with the trait, 254 caution is needed due to its high type I error rate. For a signal detected by 255 the marginal test, reanalysis in full model could be valuable. Ridge regression 256 is preferable compared to the full model without ridge regularization because 257 ridge estimator of the effect size has smaller MSE (equation (13)). Nonlinear 258 regression, which models scales properly, is recommended more than the 259 linear regression, yet the difference can be modest. We do not claim the ridge 260 model to substitute previous models. Indeed, we think none of the current 261 algorithms is superior to others in all aspects, indicating possibility for future 262 improvement.

\section{Methods}

\section{Linear regression}

266 We begin by describing the linear regressions used in previous studies. Let 267 the indexes be $h$ for a cell type, $i$ for a sample, $j$ for an omics marker (CpG 268 site or gene), $k$ for a trait that has cell-type-specific effects on marker 269 expression, and $/$ for a trait that has a uniform effect across cell types. The 270 input data is given in four matrices. The matrix $W_{h, i}$ represents cell type 271 composition. The matrices $X_{i, k}$ and $C_{i, l}$ represent the values of the traits that 272 have cell-type-specific and uniform effects, respectively. We assume the two 273 matrices are centered: $\sum_{i} X_{i, k}=\sum_{i} C_{i, l}=0$. The matrix $Y_{i, j}$ represents the 274 omics marker expression level in tissue samples.

275 The parameters we estimate are the cell-type-specific trait effect $\beta_{h, j, k}$ 276 tissue-uniform trait effect $\gamma_{j, l}$, and basal marker level $\alpha_{h, j}$ in each cell type. 277 For the remaining of the first five sections (up to "Multicollinearity of 278 interaction terms"), we focus on one marker $j$, and omit the index for 279 readability. For cell type $h$, the marker level of sample $i$ is

$$
\alpha_{h}+\sum_{k} \beta_{h, k} X_{i, k} .
$$


281 This is a representative value rather than a mean because we do not model

282 a probability distribution for cell-type-specific expression. By averaging the 283 value over cell types with weight $W_{h, i}$, and combining with the tissue-uniform 284 trait effects, we obtain the mean marker level in bulk tissue of sample $i$,

$$
\mu_{i}=\sum_{h} \alpha_{h} W_{h, i}+\sum_{h, k} \beta_{h, k} W_{h, i} X_{i, k}+\sum_{l} \gamma_{l} C_{i, l}
$$

With regards to the statistical model, we assume the error of the marker level to be normally distributed with variance $\sigma^{2}$, independently among samples, as

$$
\begin{gathered}
Y_{i}=\mu_{i}+\varepsilon_{i}, \\
\varepsilon_{i} \sim N\left(0, \sigma^{2}\right) .
\end{gathered}
$$

291 The statistical significance of all parameters is tested under the full model of linear regression,

$$
Y_{i}=\sum_{h} \alpha_{h} W_{h, i}+\sum_{h, k} \beta_{h, k} W_{h, i} X_{i, k}+\sum_{l} \gamma_{l} C_{i, l}+\varepsilon_{i}
$$

294 or its derivatives $[5,10,13]$. Alternatively, the cell-type-specific effects of 295 traits can be fitted and tested for one cell type $h$ at a time by the marginal 296 model,

$$
Y_{i}=\sum_{h^{\prime}} \alpha_{h^{\prime}} W_{h^{\prime}, i}+\sum_{k} \beta_{h, k} W_{h, i} X_{i, k}+\sum_{l} \gamma_{l} C_{i, l}+\varepsilon_{i}
$$

298 or its derivatives $[7-9,11,14]$.

\section{Nonlinear regression}

300 Aiming to simultaneously analyze cell type composition in linear scale and 301 differential expression/methylation in log/logit scale, we develop a nonlinear 302 regression model. The differential analyses are performed after applying 303 normalizing transformation. The normalizing function is the natural logarithm $304 f=\log$ for gene expression, and $f=\operatorname{logit}$ for methylation (see Background). 305 Conventional linear regression can be formulated by defining $f$ as the identity 306 function. We denote the inverse function of $f$ by $g ; g=\exp$ for gene 307 expression, and $g=$ logistic for methylation. Thus, $f$ converts from the linear 308 scale to the normalized scale, and $g$ does the opposite. 
The marker level in a specific cell type (formula (1)) is modeled in the 310 normalized scale. The level is linearized by applying function $g$, then averaged 311 over cell types with weight $W_{h, i}$, and normalized by applying function $f$. 312 Combined with the tissue-uniform trait effects, the mean normalized marker 313 level in bulk tissue of sample $i$ becomes

$$
\mu_{i}=f\left(\sum_{h} W_{h, i} g\left(\alpha_{h}+\sum_{k} \beta_{h, k} X_{i, k}\right)\right)+\sum_{l} \gamma_{l} C_{i, l} .
$$

We assume the normalized marker level to have an error that is normally distributed with variance $\sigma^{2}$, independently among samples, as

$$
\begin{aligned}
f\left(Y_{i}\right) & =\mu_{i}+\varepsilon_{i}, \\
\varepsilon_{i} & \sim N\left(0, \sigma^{2}\right) .
\end{aligned}
$$

We obtain the ordinary least squares (OLS) estimator of the parameters by minimizing the residual sum of squares,

$$
\operatorname{RSS}=\sum_{i}\left(f\left(Y_{i}\right)-\mu_{i}\right)^{2}
$$

and then estimate the error variance as

$$
\widehat{\sigma^{2}}=\frac{1}{n-p} \mathrm{RSS},
$$

324 where $n$ is the number of samples and $p$ is the number of parameters [[24], 325 section 6.3.1].

\section{Ridge regression}

327 The parameters $\beta_{h, k}$ for cell-type-specific effect cannot be estimated 328 accurately by ordinary linear regression because the regressors $W_{h, i} X_{i, k}$ in 329 equation (2) are highly correlated between cell types (see below). 330 Multicollinearity also occurs to the nonlinear case in formula (4) because of 331 local linearity. To cope with the multicollinearity, we apply ridge regression 332 with a regularization parameter $\lambda \geq 0$, and obtain the ridge estimator of the 333 parameters that minimizes

$$
\mathrm{RSS}+\lambda \sum_{h, k} \beta_{h, k}^{2}
$$


335 where the second term penalizes $\beta_{h, k}$ for taking large absolute values. The 336 ridge estimator $\widehat{\boldsymbol{\theta}}(\lambda)$ is asymptotically normally distributed (see Additional file 337 2: Supplementary note) with

$$
\operatorname{Mean}[\widehat{\boldsymbol{\theta}}(\lambda)]=Q(\lambda)^{-1} Q(0) \boldsymbol{\theta},
$$

39

$$
\begin{gathered}
\operatorname{Var}[\widehat{\boldsymbol{\theta}}(\lambda)]=\sigma^{2} Q(\lambda)^{-1}\left(\frac{\partial \boldsymbol{\mu}(\boldsymbol{\theta})}{\partial \boldsymbol{\theta}}\right)^{T}\left(\frac{\partial \boldsymbol{\mu}(\boldsymbol{\theta})}{\partial \boldsymbol{\theta}}\right) Q(\lambda)^{-1}, \\
Q(\lambda)=\left(\frac{\partial \boldsymbol{\mu}(\boldsymbol{\theta})}{\partial \boldsymbol{\theta}}\right)^{T}\left(\frac{\partial \boldsymbol{\mu}(\boldsymbol{\theta})}{\partial \boldsymbol{\theta}}\right)+\lambda\left(\begin{array}{lll}
O & O & O \\
O & I & 0 \\
O & O & O
\end{array}\right)-(f(Y)-\boldsymbol{\mu}(\boldsymbol{\theta})) \cdot\left(\frac{\partial^{2} \boldsymbol{\mu}(\boldsymbol{\theta})}{\partial \boldsymbol{\theta} \partial \boldsymbol{\theta}^{T}}\right),
\end{gathered}
$$

341 where $\boldsymbol{\mu}$ is the vector form of $\mu_{i}, \boldsymbol{\theta}$ is the vector form of the parameters $\alpha_{h}$, $342 \beta_{h, k}$ and $\gamma_{l}$ combined, $(\partial \boldsymbol{\mu} / \partial \boldsymbol{\theta})$ is the Jacobian matrix, $\left(\partial^{2} \boldsymbol{\mu} / \partial \boldsymbol{\theta} \partial \boldsymbol{\theta}^{T}\right)$ is the 343 array of Hessian matrices for $\mu_{i}$ taken over samples, and $T$ indicates matrix 344 transposition. The product of $f(Y)-\boldsymbol{\mu}(\boldsymbol{\theta})$ and the Hessian is taken by multiplying for each sample and then summing up over samples. The matrix after $\lambda$ has one only in the diagonal corresponding to $\beta_{h, k}$. The assigned value $\boldsymbol{\theta}$ is the true parameter value. By taking the expectation of $Q$, we obtain a rougher approximation [25] as

$$
\operatorname{Mean}[\widehat{\boldsymbol{\theta}}(\lambda)]=Q^{*}(\lambda)^{-1} Q^{*}(0) \boldsymbol{\theta},
$$

350

351

$$
\begin{aligned}
\operatorname{Var}[\widehat{\boldsymbol{\theta}}(\lambda)] & =\sigma^{2} \quad Q^{*}(\lambda)^{-1}\left(\frac{\partial \boldsymbol{\mu}(\boldsymbol{\theta})}{\partial \boldsymbol{\theta}}\right)^{T}\left(\frac{\partial \boldsymbol{\mu}(\boldsymbol{\theta})}{\partial \boldsymbol{\theta}}\right) Q^{*}(\lambda)^{-1}, \\
Q^{*}(\lambda) & =\mathrm{E}[Q(\lambda)]=\left(\frac{\partial \boldsymbol{\mu}(\boldsymbol{\theta})}{\partial \boldsymbol{\theta}}\right)^{T}\left(\frac{\partial \boldsymbol{\mu}(\boldsymbol{\theta})}{\partial \boldsymbol{\theta}}\right)+\lambda\left(\begin{array}{lll}
O & O & 0 \\
O & I & 0 \\
O & O & O
\end{array}\right) .
\end{aligned}
$$

352 The matrices $Q$ and $Q^{*}$ are the observed and expected Fisher matrices 353 multiplied by $\sigma^{2}$ and adapted to ridge regression, respectively.

354 Since our objective is to predict the cell-type-specific trait effects, we 355 choose the regularization parameter $\lambda$ that can minimize the mean squared 356 error (MSE) of $\beta_{h, k}$. Our methodology is based on [26]. To simplify the 357 explanation, we assume the Jacobian matrices $(\partial \boldsymbol{\mu}(\boldsymbol{\theta}) / \partial \boldsymbol{\alpha}),(\partial \boldsymbol{\mu}(\boldsymbol{\theta}) / \partial \boldsymbol{\beta})$ and $358(\partial \boldsymbol{\mu}(\boldsymbol{\theta}) / \partial \boldsymbol{\gamma})$ to be mutually orthogonal, where $\boldsymbol{\alpha}, \boldsymbol{\beta}$ and $\boldsymbol{\gamma}$ are the vector 359 forms of $\alpha_{h}, \beta_{h, k}$ and $\gamma_{l}$, respectively. Then, from formulae (11) and (12), the 360 ridge estimator $\widehat{\boldsymbol{\beta}}(\lambda)$ is asymptotically normally distributed with

$$
\operatorname{Mean}[\widehat{\boldsymbol{\beta}}(\lambda)]=\left[\left(\frac{\partial \boldsymbol{\mu}(\boldsymbol{\theta})}{\partial \boldsymbol{\beta}}\right)^{T}\left(\frac{\partial \boldsymbol{\mu}(\boldsymbol{\theta})}{\partial \boldsymbol{\beta}}\right)+\lambda I\right]^{-1}\left(\frac{\partial \boldsymbol{\mu}(\boldsymbol{\theta})}{\partial \boldsymbol{\beta}}\right)^{T}\left(\frac{\partial \boldsymbol{\mu}(\boldsymbol{\theta})}{\partial \boldsymbol{\beta}}\right) \boldsymbol{\beta},
$$




$$
\operatorname{Var}[\widehat{\boldsymbol{\beta}}(\lambda)]=\sigma^{2}\left[\left(\frac{\partial \boldsymbol{\mu}(\boldsymbol{\theta})}{\partial \boldsymbol{\beta}}\right)^{T}\left(\frac{\partial \boldsymbol{\mu}(\boldsymbol{\theta})}{\partial \boldsymbol{\beta}}\right)+\lambda I\right]^{-1}\left(\frac{\partial \boldsymbol{\mu}(\boldsymbol{\theta})}{\partial \boldsymbol{\beta}}\right)^{T}\left(\frac{\partial \boldsymbol{\mu}(\boldsymbol{\theta})}{\partial \boldsymbol{\beta}}\right)
$$

$$
\left[\left(\frac{\partial \boldsymbol{\mu}(\boldsymbol{\theta})}{\partial \boldsymbol{\beta}}\right)^{T}\left(\frac{\partial \boldsymbol{\mu}(\boldsymbol{\theta})}{\partial \boldsymbol{\beta}}\right)+\lambda I\right]^{-1},
$$

364 where the assigned values $\boldsymbol{\theta}$ and $\boldsymbol{\beta}$ are the true parameter values. We apply 365 singular value decomposition

$$
\left(\frac{\partial \boldsymbol{\mu}(\boldsymbol{\theta})}{\partial \boldsymbol{\beta}}\right)=U D V^{T},
$$

367 where $U$ and $V$ are orthogonal matrices, the columns of $V$ are $v_{1}, \cdots, v_{M}$, and 368 the diagonals of diagonal matrix $D$ are sorted $d_{1} \geq \cdots \geq d_{M} \geq 0$. The bias, variance and MSE of the ridge estimator are decomposed as

$$
\begin{aligned}
& \operatorname{Bias}[\widehat{\boldsymbol{\beta}}(\lambda)]=\mathrm{E}[\widehat{\boldsymbol{\beta}}(\lambda)-\boldsymbol{\beta}] \\
& =-\lambda\left[\left(\frac{\partial \boldsymbol{\mu}(\boldsymbol{\theta})}{\partial \boldsymbol{\beta}}\right)^{T}\left(\frac{\partial \boldsymbol{\mu}(\boldsymbol{\theta})}{\partial \boldsymbol{\beta}}\right)+\lambda I\right]^{-1} \boldsymbol{\beta} \\
& \quad=\left\{\sum_{m=1}^{M} \boldsymbol{v}_{m} \frac{-\lambda}{d_{m}{ }^{2}+\lambda} \boldsymbol{v}_{m}{ }^{T}\right\} \boldsymbol{\beta}, \\
& \operatorname{Var}[\widehat{\boldsymbol{\beta}}(\lambda)]=\sigma^{2} \sum_{m=1}^{M} \boldsymbol{v}_{m} \frac{d_{m}{ }^{2}}{\left(d_{m}{ }^{2}+\lambda\right)^{2}} \boldsymbol{v}_{m}{ }^{T}, \\
& \operatorname{MSE}[\widehat{\boldsymbol{\beta}}(\lambda)]=\mathrm{E}\left[\|\widehat{\boldsymbol{\beta}}(\lambda)-\boldsymbol{\beta}\|^{2}\right] \\
& =\|\operatorname{Bias}[\widehat{\boldsymbol{\beta}}(\lambda)]\|^{2}+\operatorname{tr}(\operatorname{Var}[\widehat{\boldsymbol{\beta}}(\lambda)]) \\
& =\sum_{m=1}^{M}\left(\frac{\lambda}{d_{m}{ }^{2}+\lambda}\right)^{2}\left(\boldsymbol{v}_{m}{ }^{T} \boldsymbol{\beta}\right)^{2}+\left(\frac{d_{m}{ }^{2}}{d_{m}{ }^{2}+\lambda}\right)^{2}\left(\frac{\sigma^{2}}{d_{m}{ }^{2}}\right) .
\end{aligned}
$$

For each $m$ in the summation of (13), the minimum of the summand is attained at $\lambda_{m}=\sigma^{2} /\left(\boldsymbol{v}_{m}{ }^{T} \boldsymbol{\beta}\right)^{2}$. To minimize $\mathrm{MSE}$, we need to find some "average" of the optimal $\lambda_{m}$ over the range of $m$. Hoerl et al. [27] proposed to take the harmonic mean $\lambda=M \sigma^{2} /\|\boldsymbol{\beta}\|^{2}$. However, if an OLS estimator $\widehat{\boldsymbol{\beta}}(0)$ 381 is plugged in, $\|\boldsymbol{\beta}\|^{2}$ is biased upwards, and $\lambda$ is biased downwards. Indeed, 382 with regards to the estimator of $1 / \sqrt{\lambda_{m}}$, we notice that

$$
\frac{1}{\sigma} \boldsymbol{v}_{m}{ }^{T} \widehat{\boldsymbol{\beta}}(0) \sim N\left(\frac{1}{\sigma} \boldsymbol{v}_{m}{ }^{T} \boldsymbol{\beta}, \frac{1}{d_{m}{ }^{2}}\right),
$$

384 where the terms with larger $m$ have larger variance. Thus, we take the 385 average of $\left(\boldsymbol{v}_{m}{ }^{T} \widehat{\boldsymbol{\beta}}(0)\right)^{2} / \sigma^{2}$, weighted by $d_{m}{ }^{2} / \sum_{m=1}^{M} d_{m}{ }^{2}$, and also subtract the upward bias as, 


$$
\kappa=\frac{1}{\sum_{m=1}^{M} d_{m}^{2}} \sum_{m=1}^{M}\left\{\frac{d_{m}{ }^{2}\left(\boldsymbol{v}_{m}{ }^{T} \widehat{\boldsymbol{\beta}}(0)\right)^{2}}{\sigma^{2}}-1\right\} .
$$

388 The weighting and subtraction were mentioned in [26], where the subtraction 389 term was dismissed, under the assumption of large effect-size $\boldsymbol{\beta}$. Since the 390 effect-size could be small in our application, we keep the subtraction term.

391 The statistic $\kappa$ can be nonpositive, and is unbiased in the sense that

$$
\mathrm{E}[\kappa]=\frac{1}{\sum_{m=1}^{M} d_{m}{ }^{2}} \sum_{m=1}^{M} \frac{d_{m}{ }^{2}\left(\boldsymbol{v}_{m}{ }^{T} \boldsymbol{\beta}\right)^{2}}{\sigma^{2}}=\frac{1}{\sum_{m=1}^{M} d_{m}{ }^{2}} \sum_{m=1}^{M} \frac{d_{m}{ }^{2}}{\lambda_{m}} .
$$

393 Our choice of regularization parameter is

$$
\lambda=\left\{\begin{array}{c}
1 / \kappa \text { if } \kappa>0, \\
d_{1}{ }^{2} \text { otherwise, }
\end{array}\right.
$$

where $d_{1}^{2}$ is taken instead of positive infinity.

\section{Implementation of omicwas package}

For each omics marker, the parameters $\boldsymbol{\alpha}, \boldsymbol{\beta}$ and $\boldsymbol{\gamma}$ (denoted in combination by $\boldsymbol{\theta}$ ) are estimated and tested by nonlinear ridge regression in the following steps. As we assume the magnitude of trait effects $\beta$ and $\gamma$ to be much smaller than that of basal marker level $\alpha$, we first fit $\alpha$ alone for numerical stability.

1. Compute OLS estimator $\widehat{\boldsymbol{\alpha}}(0)$ by minimizing formula (6) under $\boldsymbol{\beta}=\boldsymbol{\gamma}=\mathbf{0}$. Apply Wald test.

2. Calculate $\widehat{\sigma^{2}}$ by formula (7). Use it as a substitute for $\sigma^{2}$. The residual 405 degrees of freedom $n-p$ is the number of samples minus the number of parameters in $\alpha$.

3. Compute OLS estimators $\widehat{\boldsymbol{\beta}}(0)$ and $\widehat{\gamma}(0)$ by minimizing formula (6) under $\boldsymbol{\alpha}=\widehat{\boldsymbol{\alpha}}(0)$. Let $\widehat{\boldsymbol{\theta}}(0)=\left(\widehat{\boldsymbol{\alpha}}(0)^{T}, \widehat{\boldsymbol{\beta}}(0)^{T}, \widehat{\boldsymbol{\gamma}}(0)^{T}\right)^{T}$.

409 4. Apply singular value decomposition $(\partial \boldsymbol{\mu}(\widehat{\boldsymbol{\theta}}(0)) / \partial \boldsymbol{\beta})=U D V^{T}$.

4105 . Calculate $\kappa$ and then the regularization parameter $\lambda$ by formulae (14) 411 and (15).

412 6. Compute ridge estimators $\widehat{\boldsymbol{\beta}}(\lambda)$ and $\widehat{\gamma}(\lambda)$ by minimizing formula (8) under $413 \boldsymbol{\alpha}=\widehat{\boldsymbol{\alpha}}(0)$. Let $\widehat{\boldsymbol{\theta}}(\lambda)=\left(\widehat{\boldsymbol{\alpha}}(0)^{T}, \widehat{\boldsymbol{\beta}}(\lambda)^{T}, \widehat{\boldsymbol{\gamma}}(\lambda)^{T}\right)^{T}$.

414 7. Approximate the variance of ridge estimator, according to formula (10), 
by

$$
\operatorname{Var}\left[\left(\begin{array}{l}
\widehat{\boldsymbol{\beta}}(\lambda) \\
\widehat{\boldsymbol{\gamma}}(\lambda)
\end{array}\right)\right]=\widehat{\sigma^{2}} Q(\lambda)^{-1}\left(\frac{\partial \boldsymbol{\mu}(\widehat{\boldsymbol{\theta}}(\lambda))}{\partial\left(\begin{array}{l}
\boldsymbol{\beta} \\
\boldsymbol{\gamma}
\end{array}\right)}\right)^{T}\left(\frac{\partial \boldsymbol{\mu}(\widehat{\boldsymbol{\theta}}(\lambda))}{\partial\left(\begin{array}{l}
\boldsymbol{\beta} \\
\boldsymbol{\gamma}
\end{array}\right)}\right) Q(\lambda)^{-1},
$$

$$
Q(\lambda)=\left(\frac{\partial \boldsymbol{\mu}(\widehat{\boldsymbol{\theta}}(\lambda))}{\partial\left(\begin{array}{l}
\boldsymbol{\beta} \\
\boldsymbol{\gamma}
\end{array}\right)}\right)^{T}\left(\frac{\partial \boldsymbol{\mu}(\widehat{\boldsymbol{\theta}}(\lambda))}{\partial\left(\begin{array}{l}
\boldsymbol{\beta} \\
\boldsymbol{\gamma}
\end{array}\right)}\right)+\lambda\left(\begin{array}{ll}
I & 0 \\
0 & 0
\end{array}\right)-(f(Y)-\boldsymbol{\mu}(\widehat{\boldsymbol{\theta}}(\lambda))) \cdot\left(\frac{\partial^{2} \boldsymbol{\mu}(\widehat{\boldsymbol{\theta}}(\lambda))}{\partial\left(\begin{array}{l}
\boldsymbol{\beta} \\
\boldsymbol{\gamma}
\end{array}\right) \partial\left(\begin{array}{l}
\boldsymbol{\beta} \\
\boldsymbol{\gamma}
\end{array}\right)^{T}}\right) .
$$

8. Apply the "non-exact" $t$-type test [28]. For the $s$-th coordinate,

$$
\frac{\left(\begin{array}{l}
\widehat{\boldsymbol{\beta}}(\lambda) \\
\widehat{\boldsymbol{\gamma}}(\lambda)
\end{array}\right)_{s}}{\sqrt{\operatorname{Var}\left[\left(\begin{array}{c}
\widehat{\boldsymbol{\beta}}(\lambda) \\
\widehat{\gamma}(\lambda)
\end{array}\right)\right]_{s, s}}} \sim t_{n-p}
$$

$$
\text { under the null hypothesis }\left(\begin{array}{l}
\boldsymbol{\beta} \\
\gamma
\end{array}\right)_{s}=0 \text {. }
$$

421 The formula (16) is the same as a Wald test, but the test differs, because the 422 ridge estimators are not maximum-likelihood estimators. The algorithm was 423 implemented as a package for the R statistical language. We used the NL2SOL 424 algorithm of the PORT library [29] for minimization.

425 In analyses of quantitative trait locus (QTL), such as methylation QTL 426 (mQTL) and expression QTL (eQTL), an association analysis that takes the 427 genotypes of a single nucleotide polymorphism (SNP) as $X_{i, k}$ is repeated for 428 many SNPs. In order to speed up the computation, we perform rounds of 429 linear regression. First, the parameters $\widehat{\boldsymbol{\alpha}}(0)$ and $\widehat{\boldsymbol{\gamma}}(0)$ are fit by ordinary 430 linear regression under $\boldsymbol{\beta}=\mathbf{0}$, which does not depend on $X_{i, k}$. By taking the 431 residuals, we practically dispense with $\widehat{\boldsymbol{\alpha}}(0)$ and $\widehat{\gamma}(0)$ in the remaining steps. 432 Next, for $X_{i, k}$ of each SNP, $\widehat{\boldsymbol{\beta}}(0)$ is fit by ordinary linear regression under $\boldsymbol{\alpha}=$ $433 \widehat{\boldsymbol{\alpha}}(0), \boldsymbol{\gamma}=\widehat{\boldsymbol{\gamma}}(0)$. The regularization parameter $\lambda$ is computed according to 434 steps 4 and 5 above. Finally, $\widehat{\boldsymbol{\beta}}(\lambda)$ is fitted and tested by linear ridge 435 regression under $\boldsymbol{\alpha}=\widehat{\alpha}(0), \gamma=\widehat{\gamma}(0)$. 


\section{Multicollinearity of interaction terms}

437 The regressors for cell-type-specific trait effects in the full model (equation 438 (2)) are the interaction terms $W_{h, i} X_{i, k}$. To assess multicollinearity, we 439 mathematically derive the correlation coefficient between two interaction 440 terms $W_{h, i} X_{i, k}$ and $W_{h^{\prime}, i} X_{i, k}$. In this section, we treat $W_{h, i}, W_{h^{\prime}, i}$ and $X_{i, k}$ as 441 sampled instances of random variables $W_{h}, W_{h^{\prime}}$ and $X_{k}$, respectively. For 442 simplicity, we assume $W_{h}$ and $W_{h^{\prime}}$ are independent of $X_{k}$. Let $\mathrm{E}[\bullet], \operatorname{Var}[\bullet]$, $443 \operatorname{Cov}[\bullet]$ and $\operatorname{Cor}[\bullet]$ denote the expectation, variance, covariance and 444 correlation, respectively. Since $X_{k}$ is centered, $\mathrm{E}\left[W_{h} X_{k}\right]=\mathrm{E}\left[W_{h^{\prime}} X_{k}\right]=0$. The 445 correlation coefficient between interaction terms becomes

$$
\operatorname{Cor}\left[W_{h} X_{k}, W_{h^{\prime}} X_{k}\right]=\frac{\mathrm{E}\left[W_{h} X_{k} W_{h^{\prime}} X_{k}\right]}{\sqrt{\mathrm{E}\left[W_{h}{ }^{2} X_{k}{ }^{2}\right]} \sqrt{\mathrm{E}\left[W_{h^{\prime}}{ }^{2} X_{k}{ }^{2}\right]}}
$$

$$
=\frac{\mathrm{E}\left[W_{h} W_{h^{\prime}}\right]}{\sqrt{\mathrm{E}\left[W_{h}{ }^{2}\right]} \sqrt{\mathrm{E}\left[W_{h^{\prime}}{ }^{2}\right]}}
$$$$
=\frac{\operatorname{Cov}\left[W_{h}, W_{h^{\prime}}\right]+\mathrm{E}\left[W_{h}\right] \mathrm{E}\left[W_{h^{\prime}}\right]}{\sqrt{\operatorname{Var}\left[W_{h}\right]+\mathrm{E}\left[W_{h}\right]^{2}} \sqrt{\operatorname{Var}\left[W_{h^{\prime}}\right]+\mathrm{E}\left[W_{h^{\prime}}\right]^{2}}}
$$

$$
=\frac{\operatorname{Cor}\left[W_{h}, W_{h^{\prime}}\right]+\frac{\mathrm{E}\left[W_{h}\right]}{\sqrt{\operatorname{Var}\left[W_{h}\right]} \frac{\mathrm{E}\left[W_{h^{\prime}}\right]}{\sqrt{\operatorname{Var}\left[W_{h^{\prime}}\right]}}}}{\sqrt{1+\frac{\mathrm{E}\left[W_{h}\right]^{2}}{\operatorname{Var}\left[W_{h}\right]}} \sqrt{1+\frac{\mathrm{E}\left[W_{h^{\prime}}\right]^{2}}{\operatorname{Var}\left[W_{h^{\prime}}\right]}}} .
$$

450 If the ratios $\mathrm{E}\left[W_{h}\right] / \sqrt{\operatorname{Var}\left[W_{h}\right]}$ and $\mathrm{E}\left[W_{h^{\prime}}\right] / \sqrt{\operatorname{Var}\left[W_{h^{\prime}}\right]}$ are high, the correlation of

451 interaction terms approaches to one, irrespective of $\operatorname{Cor}\left[W_{h}, W_{h^{\prime}}\right]$.

\section{EWAS of rheumatoid arthritis}

453 EWAS datasets for rheumatoid arthritis were downloaded from the Gene 454 Expression Omnibus (GEO). Using the RnBeads package (version 2.2.0) [30] 455 of R, IDAT files of HumanMethylation 450 array were preprocessed by removing low quality samples and markers, by normalizing methylation level,

457 and by removing markers on sex chromosomes and outlier samples. The association of methylation level with disease status was tested with 
459 adjustment for sex, age, smoking status and experiment batch; the 460 covariates were assumed to have uniform effects across cell types. After 461 quality control, dataset GSE42861 included bulk peripheral blood leukocyte 462 data for 336 cases and 322 controls [20]. GSE131989 included sorted CD14 ${ }^{+}$ 463 monocyte data for 63 cases and 31 controls [21]. By meta-analysis of 464 GSE131989 and GSE87095 [19], we obtained sorted CD19+ B cell data for 465108 cases and 95 controls. The cell type composition of bulk samples was 466 imputed using the Houseman algorithm [31] in the GLINT software (version 467 1.0.4) [32].

468 Differential gene expression by age

469 Whole blood RNA-seq data of GTEx $v 7$ was downloaded from the GTEx 470 website [22]. Genes of low quality or on sex chromosomes were removed, 471 expression level was normalized, outlier samples were removed, and 389 472 samples were retained. The association of read count with age was tested 473 with adjustment for sex. From GEO dataset GSE56047 [23], we obtained 474 sorted $\mathrm{CD} 14^{+}$monocyte data for 1202 samples and sorted $\mathrm{CD} 4^{+} \mathrm{T}$ cell data 475 for 214 samples. The cell type composition of bulk samples was imputed using 476 the DeconCell package (version 0.1.0) [9] of R.

\section{Simulation of cell-type-specific disease association}

478 Bulk tissue sample data for case-control comparison were simulated based 479 on real data. We generated four scenarios. Each omics marker was simulated 480 independently. The mean expression level was defined for each cell type, 481 separately in cases and controls. The standard deviation (SD) was set to be 482 the same for each combination. We tested disease association specific to one 483 cell type, which we call the target cell type. In each scenario, the mean 484 expression level was set as follows.

485 A. The mean was equal for all cell types both in cases and controls (null 486 scenario).

487 B. The mean in cases was higher by 1 SD for the target cell type. Other 488 combinations had the same mean value. 
C. The mean in cases was lower by 1 SD for one non-target cell type. Other combinations had the same mean value.

491 D. The mean in cases was higher by 1 SD for the target cell type, and lower by $1 \mathrm{SD}$ for one non-target cell type. Other combinations had the same middle mean value.

494 The target and non-target cell types were randomly chosen for each marker. 495 For each sample, the cell-type-specific expression level was randomly 496 sampled from a normal distribution that was specified in the scenario. The 497 cell-type-specific expression levels were converted to the linear scale, and 498 then averaged across cell types, according to the predefined cell type 499 composition. The result becomes the bulk expression level of the sample in 500 linear scale.

501 We used the above-mentioned bulk tissue data, namely DNA methylation 502 data for 658 peripheral blood leukocyte samples (GSE42861) and gene 503 expression data for 389 whole blood samples (GTEx). We applied the same 504 simulation procedure to each dataset. The cell type composition in the original 505 data was retained for all samples. Half of the samples were randomly 506 assigned as cases, and the other half were assigned as controls. Normalizing 507 transformation (i.e., logit or log) was applied to the bulk expression data, and 508500 omics markers were randomly selected. For each marker, we measured 509 the average $\mu$ and the standard deviation $\sigma$ of the expression level. For 510 control samples, the expression level in each cell type was sampled from $511 N\left(\mu, \sigma^{2}\right)$. For case samples, the expression level in each cell type was sampled 512 from $N\left(\mu, \sigma^{2}\right), N\left(\mu+\sigma, \sigma^{2}\right)$ or $N\left(\mu-\sigma, \sigma^{2}\right)$ according to the scenario.

\section{Evaluation of statistical methods}

514 Cell-type-specific effects of traits was statistically tested by using bulk tissue 515 data as input. We applied the omicwas package with the normalizing function $516 f=\log$, logit, identity without ridge regularization (omicwas.log, 517 omicwas.logit, omicwas.identity) or under ridge regression 518 (omicwas.log.ridge, omicwas.logit.ridge, omicwas.identity.ridge). The 519 omicwas package was used also for conventional linear regression under the 520 full and marginal models. 
Among previous methods, we evaluated those that accept cell type

522 composition as input and compute test statistics for cell-type-specific

523 association. For DNA methylation data, we applied TOAST (version 1.2.0)

524 [10], CellDMC (version 2.4.0) [13] and TCA (version 1.1.0) [14]. CellDMC

525 first tests association for all combinations, and then filters out those not

526 differentially methylated. We took all of the initial results as

527 CellDMC.unfiltered; in CelIDMC.filtered, Z-score was set to zero for those

528 filtered out. For gene expression data, we applied TOAST and cSSAM (version

529 1.4) [5]. For csSAM, we either fitted all cell types together or one cell type at

530 a time, and denoted the results as csSAM.Im and csSAM.monovariate,

531 respectively. The csSAM method is applicable to binomial traits but not to

532 quantitative traits.

533 For simulated data, we adopted the nominal significance level $P<0.05$

534 (two-sided). In scenario $B$, the power was defined as the frequency of Z-score $535>1.96$.

536 For the association with rheumatoid arthritis and age, "true" association 537 was determined from the measurements in physically sorted blood cells, 538 under the nominal significance level $\mathrm{P}<0.05$ (two-sided). The significant 539 markers were "up-regulated" (in rheumatoid arthritis cases or elders) or 540 "down-regulated." For a set of differentially expressed markers in a cell type 541 (e.g., up-regulated in monocytes), the prediction performance of an 542 algorithm was measured by the area under the curve (AUC) of receiver 543 operating characteristic (ROC). Standard error of AUC was computed by the 544 jackknife estimator by splitting the markers into 100 groups by chromosomal 545 position. The relative performance of an algorithm was evaluated by its AUC $546-0.5$ divided by that for the best algorithm in each scenario.

\section{Supplementary information}

549 Additional file 1: Table S1. Blood cell type proportion in Tsimane 550 Amerindians, Caucasians and Hispanics.

551 Additional file 2: Supplementary note. Asymptotic distribution of ridge 552 estimator. 


\section{Abbreviations}

554 AUC : area under the curve, eQTL: expression QTL, EWAS: epigenome-wide 555 association study, GEO: Gene Expression Omnibus, mQTL: methylation QTL, 556 MSE: mean squared error, OLS: ordinary least squares, QTL: quantitative trait 557 locus, ROC: receiver operating characteristic, SD: standard deviation, SNP: 558 single nucleotide polymorphism

\section{Declarations}

\section{Ethics approval and consent to participate}

561 Not applicable.

\section{Consent for publication}

563 Not applicable.

\section{Availability of data and materials}

565 The datasets generated and analyzed during the current study are available 566 in the figshare repository, https://dx.doi.org/10.6084/m9.figshare. 10718282

\section{Competing interests}

568 The authors declare that they have no competing interests.

\section{Funding}

570 This work was supported by JSPS KAKENHI [grant number JP16K07218] and

571 by the NCGM Intramural Research Fund [grant numbers 19A2004, 20A1013].

572 The funding body had no role in the design and collection of the study, 573 experiments, analyses and interpretations of data, and in writing the 574 manuscript.

\section{Author's contributions}

576 FT developed the methodology, wrote the software, implemented the study, 577 and wrote the manuscript. NK revised the manuscript. All authors read and 578 approved the final manuscript. 


\section{Acknowledgements}

580 Not applicable.

581

\section{References}

583 1. Teschendorff $A E$, Zheng SC. Cell-type deconvolution in epigenome-wide

584 association studies: a review and recommendations. Epigenomics.

585 2017;9:757-68.

586 2. Sturm G, Finotello F, Petitprez F, Zhang JD, Baumbach J, Fridman WH, et

587 al. Comprehensive evaluation of transcriptome-based cell-type

588 quantification methods for immuno-oncology. Bioinformatics.

$5892019 ; 35: i 436-45$.

590 3. Ghosh D. Mixture models for assessing differential expression in complex

591 tissues using microarray data. Bioinformatics. 2004;20:1663-9.

592 4. Stuart RO, Wachsman W, Berry CC, Wang-Rodriguez J, Wasserman L, 593 Klacansky I, et al. In silico dissection of cell-type-associated patterns of 594 gene expression in prostate cancer. Proc Natl Acad Sci USA. National

595 Academy of Sciences; 2004;101:615-20.

596 5. Shen-Orr SS, Tibshirani R, Khatri P, Bodian DL, Staedtler F, Perry NM, et 597 al. Cell type-specific gene expression differences in complex tissues. Nat 598 Meth. Nature Publishing Group; 2010;7:287-9.

599 6. Erkkilä T, Lehmusvaara S, Ruusuvuori P, Visakorpi T, Shmulevich I, 600 Lähdesmäki H. Probabilistic analysis of gene expression measurements from 601 heterogeneous tissues. Bioinformatics. 2010;26:2571-7.

602 7. Kuhn A, Thu D, Waldvogel HJ, Faull RLM, Luthi-Carter R. Population603 specific expression analysis (PSEA) reveals molecular changes in diseased 604 brain. Nat Meth. Nature Publishing Group; 2011;8:945-7.

605 8. Westra H-J, Arends D, Esko T, Peters MJ, Schurmann C, Schramm K, et 606 al. Cell Specific eQTL Analysis without Sorting Cells. Pastinen T, editor. PLoS 
607 Genet. Public Library of Science; 2015;11:e1005223-17.

608 9. Aguirre-Gamboa R, de Klein N, di Tommaso J. Deconvolution of bulk 609 blood eQTL effects into immune cell subpopulations. bioRxiv. 2019.

610 10. Li Z, Wu Z, Jin P, Wu H. Dissecting differential signals in high-

611 throughput data from complex tissues. Hancock J, editor. Bioinformatics.

612 2019;35:3898-905.

613 11. Montaño CM, Irizarry RA, Kaufmann WE, Talbot K, Gur RE, Feinberg AP, 614 et al. Measuring cell-type specific differential methylation in human brain 615 tissue. Genome Biol. BioMed Central; 2013;14:R94-9.

616 12. White $N$, Benton $M$, Kennedy $D$, Fox A, Griffiths $L$, Lea $R$, et al.

617 Accounting for cell lineage and sex effects in the identification of cell618 specific DNA methylation using a Bayesian model selection algorithm.

619 Sawalha AH, editor. PLoS ONE. 2017;12:e0182455-18.

620 13. Zheng SC, Breeze CE, Beck S, Teschendorff AE. Identification of 621 differentially methylated cell types in epigenome-wide association studies. 622 Nat Meth. Nature Publishing Group; 2018;15:1059-66.

623 14. Rahmani E, Schweiger R, Rhead B, Criswell LA, Barcellos LF, Eskin E, et 624 al. Cell-type-specific resolution epigenetics without the need for cell sorting 625 or single-cell biology. Nature Communications. Nature Publishing Group; $6262019 ; 10: 3417-11$.

627 15. Cobos FA, Vandesompele J, Mestdagh P. Computational deconvolution 628 of transcriptomics data from mixed cell populations. Bioinformatics. $6292018 ; 34: 1969-79$.

630 16. Hoyle DC, Rattray M, Jupp R, Brass A. Making sense of microarray data 631 distributions. Bioinformatics. 2002;18:576-84.

632 17. Du $P$, Zhang $X$, Huang $C-C$, Jafari N, Kibbe WA, Hou L, et al.

633 Comparison of Beta-value and $M$-value methods for quantifying methylation 
634 levels by microarray analysis. BMC Bioinformatics. BioMed Central;

635 2010;11:1-9.

636 18. Aiken LS, West SG. Multiple Regression: Testing and Interpreting

637 Interactions. Sage Publications; 1991.

638 19. Julià $A$, Absher $D$, López-Lasanta $M$, Palau N, Pluma A, Waite Jones $L$, et 639 al. Epigenome-wide association study of rheumatoid arthritis identifies

640 differentially methylated loci in B cells. Hum Mol Genet. 2017;26:2803-11.

641 20. Liu Y, Aryee MJ, Padyukov L, Fallin MD, Hesselberg E, Runarsson A, et 642 al. Epigenome-wide association data implicate DNA methylation as an 643 intermediary of genetic risk in rheumatoid arthritis. Nat Biotechnol. Nature 644 Publishing Group; 2013;31:142-7.

645 21. Rhead B, Holingue C, Cole M, Shao X, Quach HL, Quach D, et al. 646 Rheumatoid Arthritis Naive T Cells Share Hypermethylation Sites With 647 Synoviocytes. Arthritis \& Rheumatology. John Wiley \& Sons, Ltd; $648 \quad 2017 ; 69: 550-9$.

649 22. GTEx Consortium. Genetic effects on gene expression across human 650 tissues. Nature. Nature Publishing Group; 2017;550:204-13.

651 23. Reynolds LM, Taylor JR, Ding J, Lohman K, Johnson C, Siscovick D, et 652 al. Age-related variations in the methylome associated with gene expression 653 in human monocytes and T cells. Nature Communications. 2014;5:5366.

654 24. Riazoshams H, Midi H, Ghilagaber G. Robust Nonlinear Regression: with 655 Applications using R. John Wiley \& Sons; 2019.

656 25. Lim C. Robust ridge regression estimators for nonlinear models with 657 applications to high throughput screening assay data. Statist. Med. $658 \quad 2014 ; 34: 1185-98$.

659 26. Lawless JF, Wang P. A simulation study of ridge and other regression 660 estimators. Communications in Statistics - Theory and Methods. 
$661 \quad 1976 ; 5: 307-23$.

662 27. Hoerl AE, Kannard RW, Baldwin KF. Ridge regression: some simulations. 663 Communications in Statistics - Theory and Methods. 1975;4:105-23.

664 28. Halawa AM, Bassiouni El MY. Tests of regression coefficients under ridge 665 regression models. Journal of Statistical Computation and Simulation. $6662000 ; 65: 341-56$.

667 29. Dennis JE, Gay DM, Welsch RE. An adaptive nonlinear least-squares 668 algorithm. ACM Transactions on Mathematical Software. 1981;7:348-68.

669 30. Müller F, Scherer M, Assenov Y, Lutsik P, Walter J, Lengauer T, et al. 670 RnBeads 2.0: comprehensive analysis of DNA methylation data. Genome 671 Biol. BioMed Central; 2019;20:55-12.

672 31. Houseman EA, Accomando WP, Koestler DC, Christensen BC, Marsit CJ, 673 Nelson $\mathrm{HH}$, et al. DNA methylation arrays as surrogate measures of cell 674 mixture distribution. BMC Bioinformatics. 2012;13:86.

675 32. Rahmani E, Yedidim R, Shenhav L, Schweiger R, Weissbrod O, Zaitlen $676 \mathrm{~N}$, et al. GLINT: a user-friendly toolset for the analysis of high-throughput 677 DNA-methylation array data. Hancock JM, editor. Bioinformatics. 678 2017;33:1870-2. 
TABLES

681

Table 1A Blood cell type proportion in rheumatoid arthritis dataset

\begin{tabular}{cccccccc}
\hline Cell type & Neu & CD4+T & CD8+T & NK & Mono & Bcells & Eos \\
\hline Mean & 0.59 & 0.10 & 0.08 & 0.08 & 0.07 & 0.07 & 0.01 \\
SD & 0.11 & 0.06 & 0.05 & 0.04 & 0.02 & 0.03 & 0.02 \\
Mean/SD & 5.3 & 1.8 & 1.6 & 1.9 & 3.2 & 2.6 & 0.4 \\
\hline
\end{tabular}

Table 1B Correlation between blood cell type proportion and rheumatoid arthritis $\left(\mathrm{X}_{\mathrm{k}}\right)$

\begin{tabular}{cccccccccc}
\hline$r$ & & Neu & CD4+T & CD8+T & NK & Mono & Bcells & Eos & $\mathbf{X}_{\mathbf{k}}=$ Disease \\
\cline { 1 - 7 } Neu & & 1 & -0.68 & -0.60 & -0.46 & -0.06 & -0.49 & -0.48 & 0.44 \\
CD4+T & -0.68 & 1 & 0.14 & 0.05 & -0.17 & 0.38 & 0.26 & -0.33 \\
CD8+T & -0.60 & 0.14 & 1 & 0.08 & -0.05 & 0.19 & 0.13 & -0.27 \\
NK & -0.46 & 0.05 & 0.08 & 1 & -0.04 & 0.01 & 0.11 & -0.27 \\
Mono & -0.06 & -0.17 & -0.05 & -0.04 & 1 & -0.17 & 0.05 & 0.10 \\
Bcells & -0.49 & 0.38 & 0.19 & 0.01 & -0.17 & 1 & 0.11 & -0.22 \\
Eos & -0.48 & 0.26 & 0.13 & 0.11 & 0.05 & 0.11 & 1 & -0.10 \\
\hline
\end{tabular}

Table 1C Correlation between interaction terms

\begin{tabular}{|c|c|c|c|c|c|c|c|}
\hline$r$ & Neu$^{*} \mathbf{X}_{\mathbf{k}}$ & CD4+T* $\mathbf{X}_{\mathbf{k}}$ & CD8+T $* X_{k}$ & $\mathbf{N K} * \mathbf{X}_{\mathbf{k}}$ & Mono $^{*} \mathbf{X}_{\mathrm{k}}$ & Bcells* $\mathbf{X}_{\mathbf{k}}$ & $\operatorname{Eos}^{*} \mathbf{X}_{\mathbf{k}}$ \\
\hline $\mathbf{N e u}^{*} \mathbf{X}_{\mathbf{k}}$ & 1 & 0.83 & 0.80 & 0.85 & 0.93 & 0.90 & 0.27 \\
\hline CD4+T* $\mathbf{X}_{\mathrm{k}}$ & 0.83 & 1 & 0.78 & 0.78 & 0.83 & 0.88 & 0.42 \\
\hline CD8+T*X & 0.80 & 0.78 & 1 & 0.77 & 0.82 & 0.83 & 0.35 \\
\hline $\mathbf{N K} * \mathbf{X}_{\mathbf{k}}$ & 0.85 & 0.78 & 0.77 & 1 & 0.85 & 0.83 & 0.35 \\
\hline Mono $^{*} \mathbf{X}_{\mathbf{k}}$ & 0.93 & 0.83 & 0.82 & 0.85 & 1 & 0.88 & 0.35 \\
\hline Bcells $* \mathbf{X}_{\mathbf{k}}$ & 0.90 & 0.88 & 0.83 & 0.83 & 0.88 & 1 & 0.36 \\
\hline $\operatorname{Eos}^{*} \mathbf{X}_{\mathbf{k}}$ & 0.27 & 0.42 & 0.35 & 0.35 & 0.35 & 0.36 & 1 \\
\hline
\end{tabular}

Neu, neutrophils; Mono, monocytes; Eos, eosinophils. 
Table 2A Blood cell type proportion in GTEx dataset

\begin{tabular}{ccccccc}
\hline Cell type & Gran & CD4+T & CD8+T & Mono & NK & Bcells \\
\hline Mean & 0.53 & 0.22 & 0.10 & 0.07 & 0.05 & 0.03 \\
SD & 0.037 & 0.020 & 0.013 & 0.004 & 0.012 & 0.003 \\
Mean/SD & 14.4 & 10.7 & 7.7 & 16.6 & 4.3 & 8.3 \\
\hline
\end{tabular}

Table 2B Correlation between blood cell type proportion and age $\left(\mathrm{X}_{\mathrm{k}}\right)$

\begin{tabular}{|c|c|c|c|c|c|c|c|}
\hline$r$ & Gran & CD4+T & CD8+T & Mono & NK & Bcells & $X_{k}=$ Age \\
\hline Gran & 1 & -0.89 & -0.83 & 0.56 & -0.76 & -0.41 & -0.23 \\
\hline CD4+T & -0.89 & 1 & 0.59 & -0.64 & 0.50 & 0.51 & 0.14 \\
\hline CD8+T & -0.83 & 0.59 & 1 & -0.40 & 0.59 & 0.15 & 0.15 \\
\hline Mono & 0.56 & -0.64 & -0.40 & 1 & -0.44 & -0.42 & 0.02 \\
\hline NK & -0.76 & 0.50 & 0.59 & -0.44 & 1 & 0.13 & 0.31 \\
\hline Bcells & -0.41 & 0.51 & 0.15 & -0.42 & 0.13 & 1 & -0.03 \\
\hline
\end{tabular}

Table 2C Correlation between interaction terms

\begin{tabular}{|c|c|c|c|c|c|c|}
\hline$r$ & $\operatorname{Gran}^{*} \mathbf{X}_{\mathbf{k}}$ & $\mathbf{C D} 4+\mathbf{T}^{*} \mathbf{X}_{\mathbf{k}}$ & $\mathbf{C D 8}+\mathbf{T}^{*} \mathbf{X}_{\mathbf{k}}$ & Mono* $\mathbf{X}_{\mathbf{k}}$ & $\mathbf{N K} * \mathbf{X}_{\mathbf{k}}$ & Bcells* $\mathbf{X}_{\mathbf{k}}$ \\
\hline Gran* $\mathbf{X}_{\mathbf{k}}$ & 1 & 0.99 & 0.98 & 1.00 & 0.96 & 0.99 \\
\hline CD4+T* $X_{k}$ & 0.99 & 1 & 1.00 & 0.99 & 0.98 & 1.00 \\
\hline $\mathrm{CD8}+\mathrm{T}^{*} \mathrm{X}_{\mathrm{k}}$ & 0.98 & 1.00 & 1 & 0.99 & 0.98 & 0.99 \\
\hline Mono $^{*} \mathbf{X}_{\mathbf{k}}$ & 1.00 & 0.99 & 0.99 & 1 & 0.96 & 0.99 \\
\hline $\mathbf{N K} * \mathbf{X}_{\mathbf{k}}$ & 0.96 & 0.98 & 0.98 & 0.96 & 1 & 0.97 \\
\hline Bcells $* \mathbf{X}_{\mathrm{k}}$ & 0.99 & 1.00 & 0.99 & 0.99 & 0.97 & 1 \\
\hline
\end{tabular}

Gra, granulocytes; Mono, monocytes. 


\section{FIGURE LEGENDS}

\section{Figure 1}

687 Contour plot of the correlation coefficient between interaction terms $W_{h} X_{k}$ 688 and $W_{h^{\prime}} X_{k} . W_{h}$ and $W_{h^{\prime}}$ represent proportions of cell types $h$ and $h^{\prime}$, and $X_{k}$ 689 represents the value of trait $k$. For this plot, we assume the ratios $690 \mathrm{E}\left[W_{h}\right] / \operatorname{SD}\left[W_{h}\right]$ and $\mathrm{E}\left[W_{h^{\prime}}\right] / \mathrm{SD}\left[W_{h^{\prime}}\right]$ to be equal. As the ratio increases $1.5,2$ to 6913 , the correlation coefficient raises $>0.5,>0.7$ to $>0.8$, over most range of $692 \operatorname{Cor}\left[W_{h}, W_{h^{\prime}}\right]$. SD stands for standard deviation.

\section{Figure 2}

694 Detection of cell-type-specific association in simulated data for DNA 695 methylation. (A), (B), (C) and (D) correspond to the respective scenarios. 696 Results from different algorithms are aligned horizontally. Vertical axis 697 indicates the Z-score for the disease effect (cases vs controls) specific to the 698 target cell type. Points are colored according to the target cell type. The 699 middle bar of the box plot indicates the median, and the lower and upper 700 hinges correspond to the first and third quartiles. The whiskers extend to the 701 value no further than $1.5 *$ inter-quartile range from the hinges. Neu, 702 neutrophils; Mono, monocytes; Eos, eosinophils.

\section{Figure 3}

704 Detection of cell-type-specific association in simulated data for gene 705 expression. (A), (B), (C) and (D) correspond to the respective scenarios. 706 Results from different algorithms are aligned horizontally. Vertical axis 707 indicates the Z-score for the disease effect (cases vs controls) specific to the 708 target cell type. Points are colored according to the target cell type. The 709 middle bar of the box plot indicates the median, and the lower and upper 710 hinges correspond to the first and third quartiles. The whiskers extend to the 711 value no further than $1.5 *$ inter-quartile range from the hinges. Gra, 712 granulocytes; Mono, monocytes. 


\section{Figure 4}

714 Performance of cell-type-specific association prediction. For rheumatoid 715 arthritis association of DNA methylation in monocytes (A) and B cells (B); 716 Age association of gene expression in $C D 4^{+} T$ cells (C) and monocytes (D). 717 The prediction is evaluated separately for up-regulated and down-regulated 718 markers. The AUC of ROC and its 95\% confidence interval are plotted for each 719 statistical algorithm. 


\section{Figures}

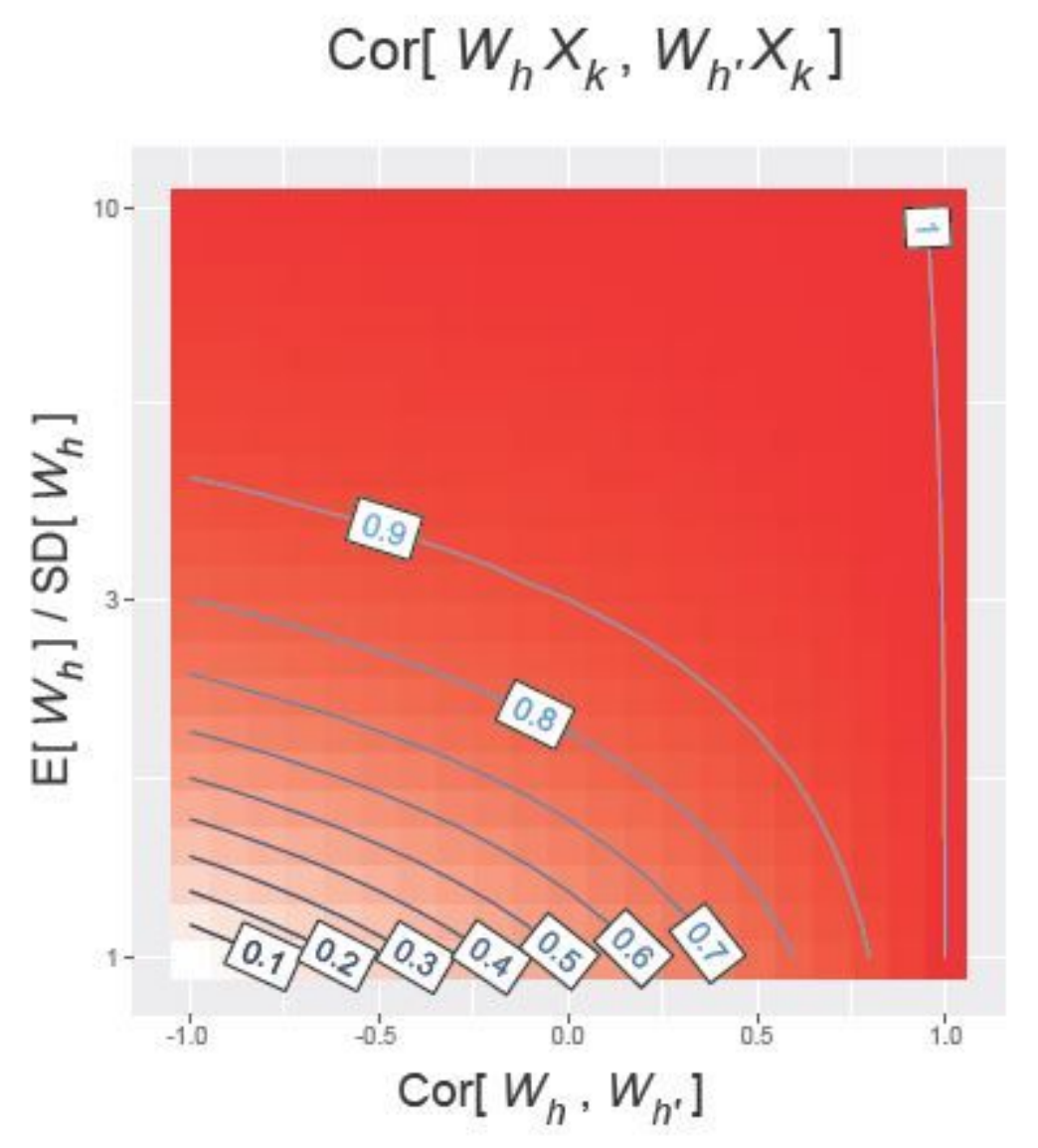

Figure 1

Contour plot of the correlation coefficient between interaction terms $W \_h X \_k$ and $W \_\left(h^{\wedge^{\prime}}\right) \mathrm{X} \_k$. W_h and $W_{-}\left(h^{\wedge^{\prime}}\right)$ represent proportions of cell types $h$ and $h^{\wedge^{\prime}}$, and $X \_k$ represents the value of trait $k$. For this plot, we assume the ratios "E" [W_h ]/"SD" [W_h ] and "E" [W_( $\left.\left.\mathrm{h}^{\wedge^{\prime}}\right)\right] /$ "SD" [W_( $\left.\left.\mathrm{h}^{\wedge^{\prime}}\right)\right]$ to be equal. As the ratio increases $1.5,2$ to 3 , the correlation coefficient raises $>0.5,>0.7$ to $>0.8$, over most range of "Cor" $\left[\mathrm{W} \_\mathrm{h}, \mathrm{W} \_\left(\mathrm{h}^{\wedge^{\prime}}\right)\right]$. SD stands for standard deviation. 
(A)
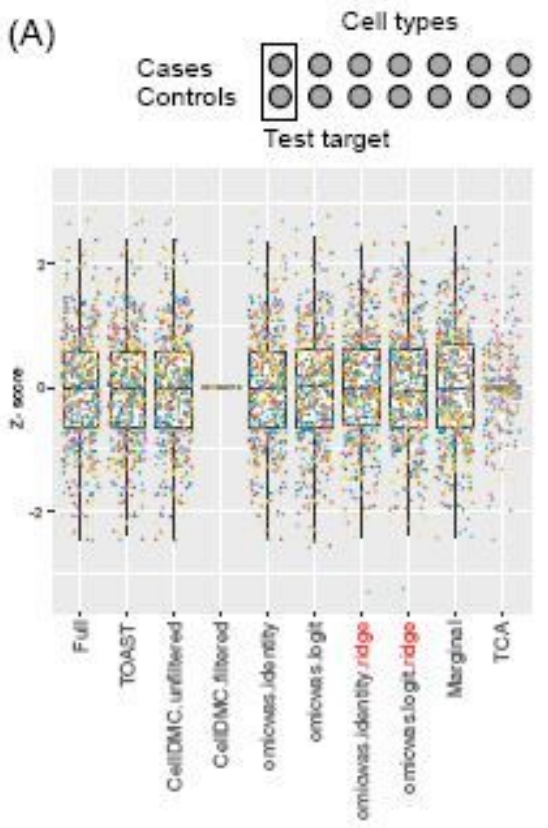

(C)
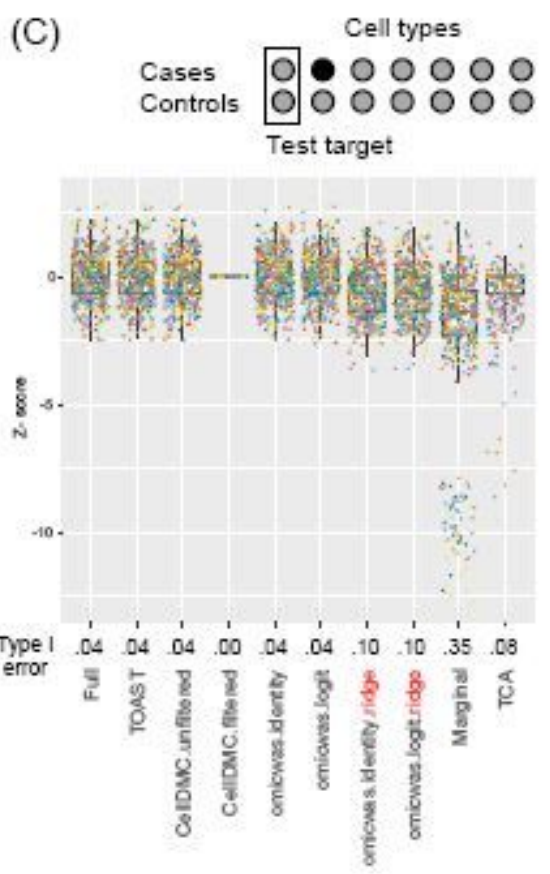

(B)
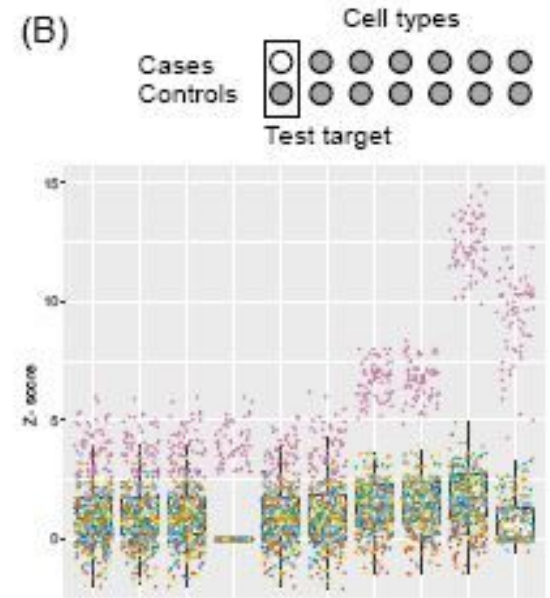

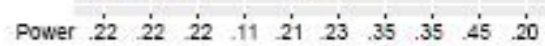

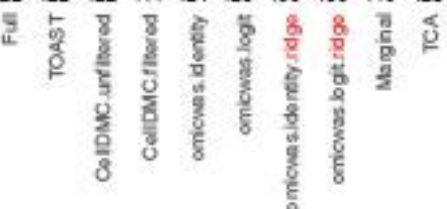

(D)
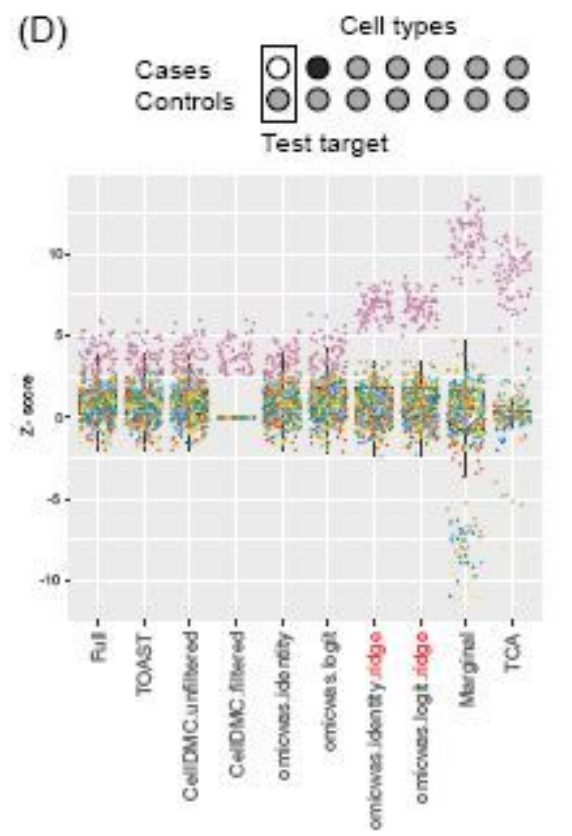

Cell type

Neu

$\mathrm{CD} 4+\mathrm{T}$

$\mathrm{CDB}+\mathrm{T}$

NK

Mono

Bcells

Eos

\section{Figure 2}

Detection of cell-type-specific association in simulated data for DNA methylation. (A), (B), (C) and (D) correspond to the respective scenarios. Results from different algorithms are aligned horizontally. Vertical axis indicates the Z-score for the disease effect (cases vs controls) specific to the target cell type. Points are colored according to the target cell type. The middle bar of the box plot indicates the median, and the lower and upper hinges correspond to the first and third quartiles. The whiskers extend to the value no further than 1.5 * inter-quartile range from the hinges. Neu, neutrophils; Mono, monocytes; Eos, eosinophils. 
(A)
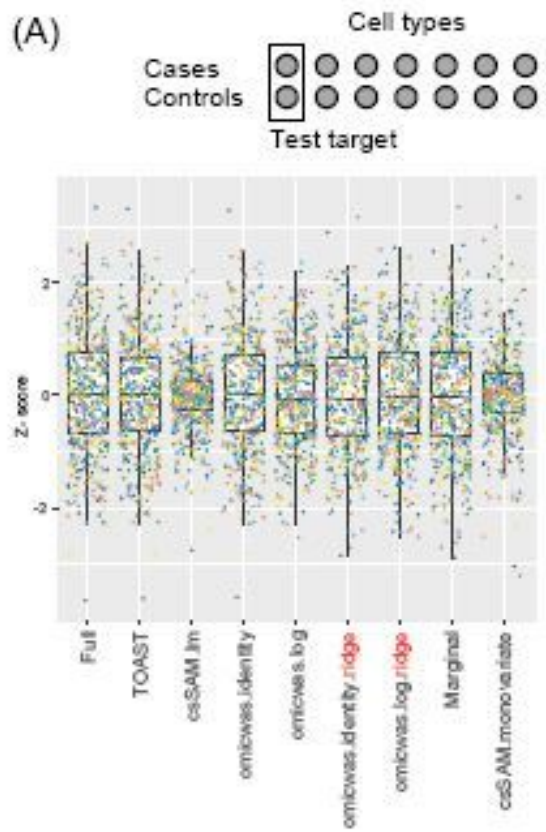

(C)
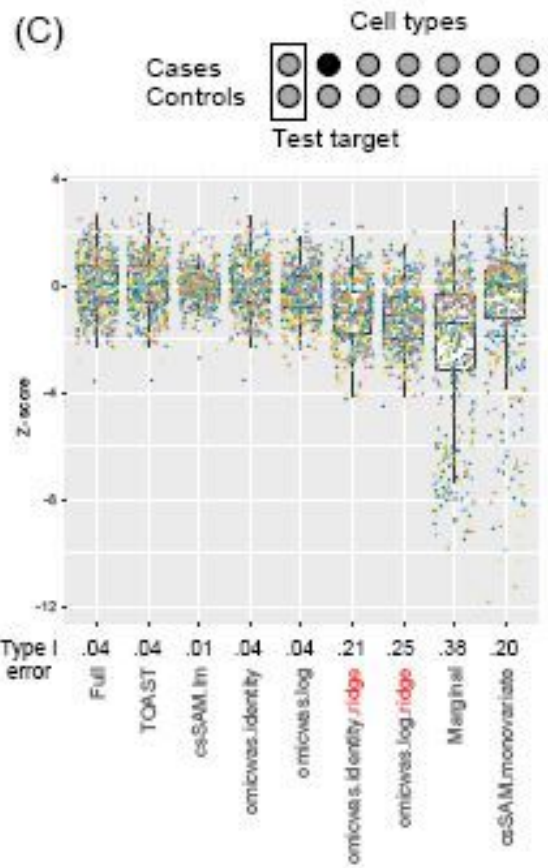

(B)
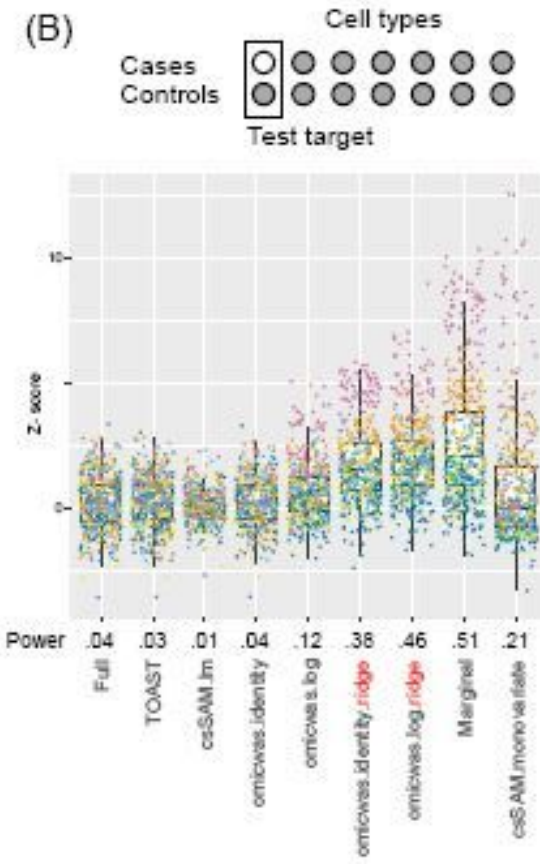

(D)
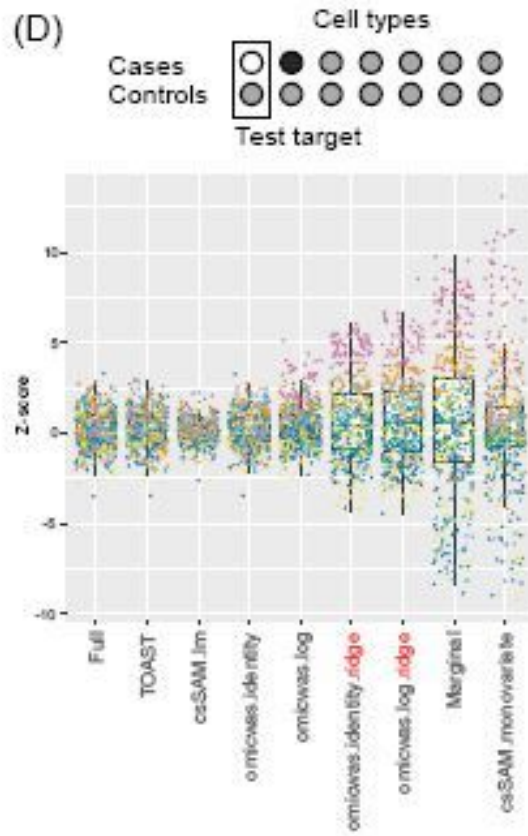

Cell type

Gran

CD4+T

CD8+T

NK

Mono

Bcells

\section{Figure 3}

Detection of cell-type-specific association in simulated data for gene expression. (A), (B), (C) and (D) correspond to the respective scenarios. Results from different algorithms are aligned horizontally. Vertical axis indicates the Z-score for the disease effect (cases vs controls) specific to the target cell type. Points are colored according to the target cell type. The middle bar of the box plot indicates the median, and the lower and upper hinges correspond to the first and third quartiles. The whiskers extend to the value no further than 1.5 * inter-quartile range from the hinges. Gra, granulocytes; Mono, monocytes. 
(A) Rheumatoid arthritis association in Monocytes

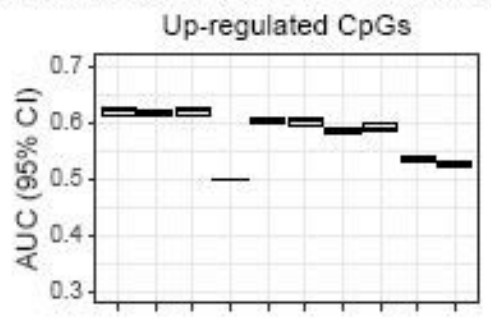

Down-regulated $\mathrm{CpGs}$

(B) Rheumatoid arthritis association in B cells
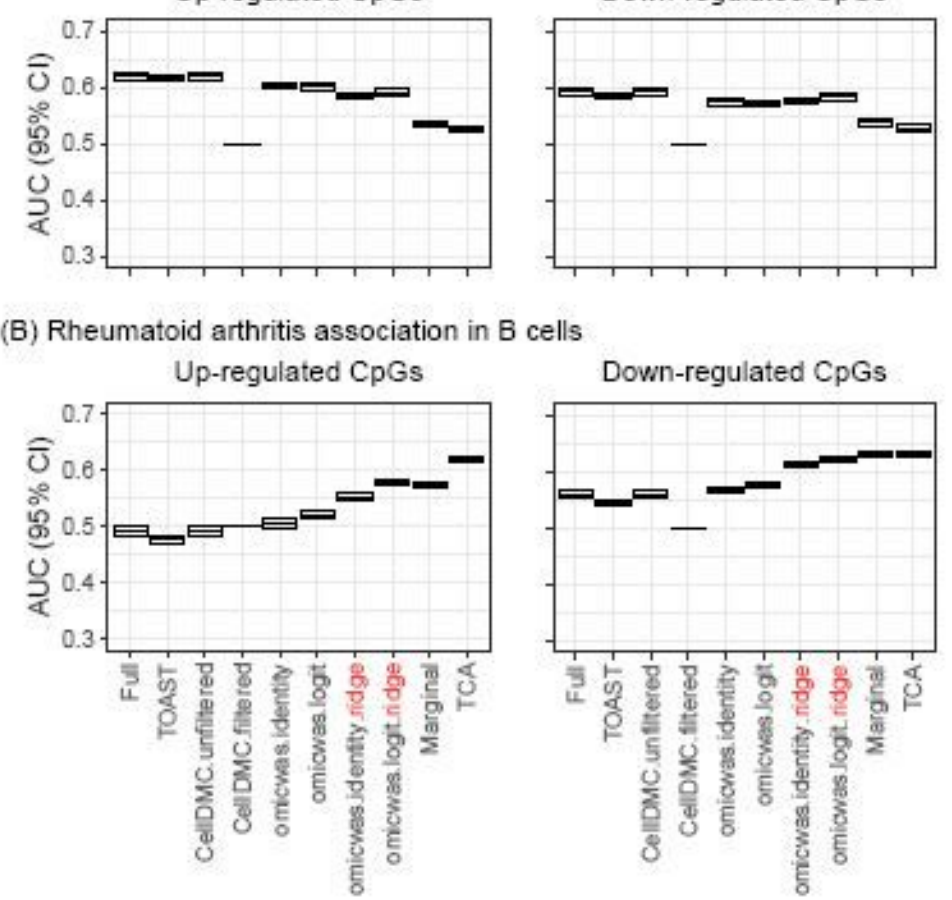

(C) Age association in CD4+ T cells
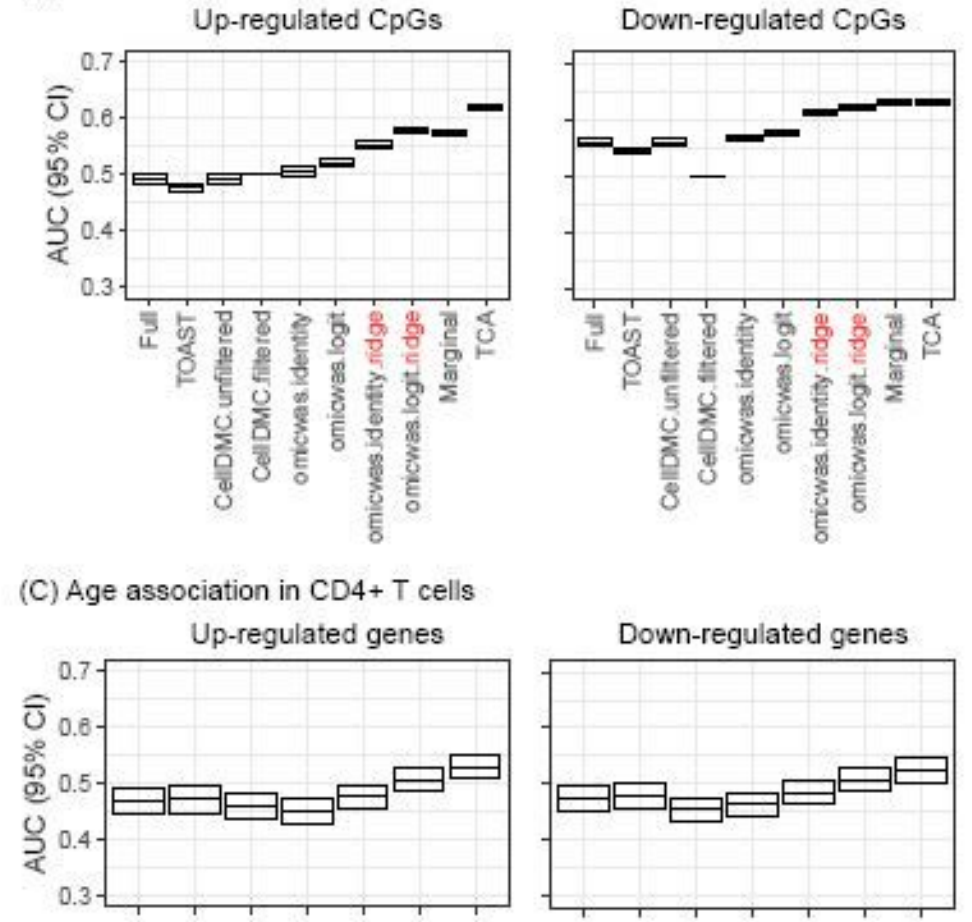

(D) Age association in Monocytes
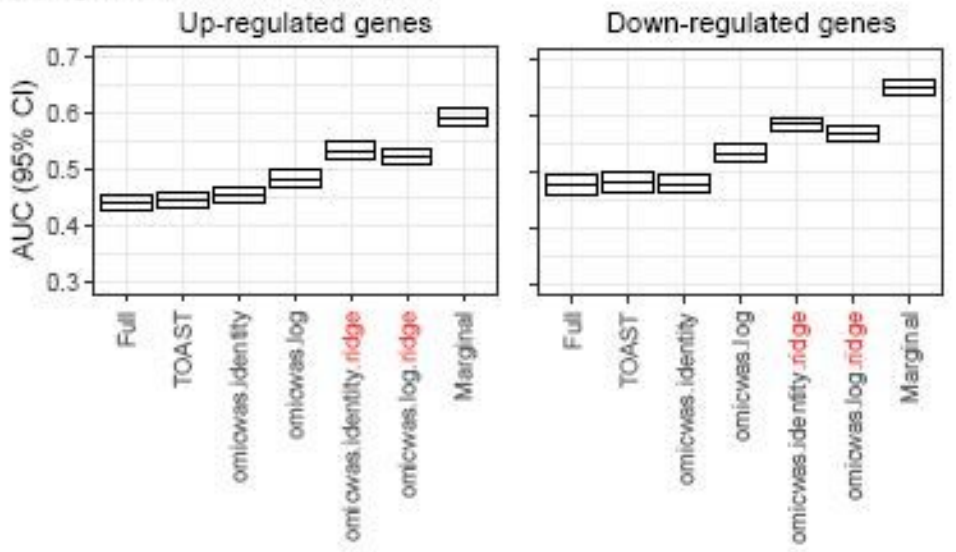

\section{Figure 4}

Performance of cell-type-specific association prediction. For rheumatoid arthritis association of DNA methylation in monocytes (A) and B cells (B); Age association of gene expression in CD4+ T cells (C) and monocytes (D). The prediction is evaluated separately for up-regulated and down-regulated markers. The AUC of ROC and its $95 \%$ confidence interval are plotted for each statistical algorithm. 


\section{Supplementary Files}

This is a list of supplementary files associated with this preprint. Click to download.

- SuppNote.pdf

- Tables1.xls 